\title{
Lo singular como alternativa conceptual y metodológica para la construcción socioespacial de ciudad*
}

\begin{tabular}{|l|l|}
\hline Fecha de recepción: 15 de noviembre de 2013 & Fecha de aceptación: 14 de enero de 2014 Disponible en línea: 30 de julio de 2014 \\
Jaime Hernández García \\
\hline Doctor en Arquitectura y Urbanismo & Profesor del Departamento de Estética, Facultad de Arquitectura y Diseño \\
\hline Pontificia Universidad Javeriana & hernandez.j@javeriana.edu.co \\
\hline
\end{tabular}

Edilsa Rojas Sánchez

\begin{tabular}{l|l} 
Psicóloga & Profesora del Departamento de Estética, Facultad de Arquitectura y Diseño
\end{tabular}

Pontificia Universidad Javeriana de Bogotá

edilsa.rojas@javeriana.edu.co

Resumen El artículo propone argumentar la pertinencia del concepto de singularidad que surge entre los espacios intersticiales de segmentos conceptuales opuestos como los de formal/ informal, legal/ilegal, centro/periferia y desplazar esta radicalidad, para promover campos teóricos alternativos que faciliten la resignificación de las condiciones socioespaciales de la ciudad en todas sus formas. Además, sugiere el desarrollo de una herramienta metodológica que se ha denominado mapeo de la singularidad que, al partir de los planes de composición planteados por Deleuze y Guattari (1997), interactúan y establecen líneas de fuerza determinantes en el tiempo y el espacio y dejan ver la movilidad social y espacial. Lo anterior se discutirá respecto a un sector popular de Bogotá, Suba-Tibabuyes y se explorarán las potencialidades de esta alternativa conceptual y metodológica.

Palabras clave Informalidad; singularidad; mapeo; fragmento; trayecto

Artículo resultado de investigación científicas y tecnológica. Titulada"Visiones alternas de ciudad: complejidad, sostenibilidad y cotidianidad". Financiado por la Pontificia Universidad Javeriana, proyecto registrado con ID 004985.

Cómo citar este artículo: Hernández-García, J. y Rojas-Sánchez, E. (2014). Lo singular como alternativa conceptual y metodológica para la construcción socioespacial de ciudad. Cuadernos de Vivienda y Urbanismo, 7(14), 184-201. http://dx.doi.org/10.11144/Javeriana.CVU7-14.sacm 


\section{The Singular as a Conceptual and Methodological Alternative for the Socio-Spatial Construction of the City}

Abstract This paper discusses the concept of singularity, which emerges within the opposite binaries of formal/informal, legal/illegal, center/periphery, aiming to overcome this rationality in order to promote theoretical alternatives that make possible to re-signify the socio-spatial characteristics of the city in all its forms. It also suggests a methodological tool called the singularity mapping, from the idea of the composition plans developed by Deleuze \& Guattari (1997), which interact and contribute to create strength lines of time and space to make visible the social and spatial mobility. The informal sector Suba-Tibabuyes will provide the empirical case to discuss the potentials of this conceptual and methodological alternative.

Keywords Informality; singularity; mapping; fragment; journey

\section{0 singular como alternativa conceitual e metodológica para a construção socioespacial de cidade}

Resumo $\mathrm{O}$ artigo visa argumentar a pertinência do conceito de singularidade que surge entre os espaços intersticiais de segmentos conceituais opostos como são formal/informal, legal/ ilegal, centro/periferia e deslocar esta radicalidade para promover campos teóricos alternativos que facilitem a ressignificação das condiçóes socioespaciais da cidade em todas suas formas. Aliás, sugere o desenvolvimento de ferramenta metodológica nomeada de mapeamento da singularidade que, ao partir dos planos de composiçáo levantados por Deleuze e Guattari (1997), interagem e estabelecem linhas de força determinantes no tempo e espaço e permitem ver a mobilidade social e espacial. Isso vai se discutir em relação a um sector popular de Bogotá, Suba-Tibabuyes e as potencialidades desta alternativa conceitual e metodológica vão ser exploradas.

Palavras chave Informalidade; singularidade; mapeamento; fragmento; trajeto 


\section{Introducción}

Las percepciones actuales sobre lo urbano (espacio-tiempo) y lo social (cultural-económico) tratan de superar los dualismos establecidos por una filosofía heredada de principios rígidos espaciales grecorromanos, ético-morales cristianos y concepciones capitalistas económicas neoliberales y corporativas, todos ellos incluidos en lo que se ha denominado filosofía o sistema de pensamiento occidental. La posición de establecer categorías duales como centro/periferia, legal/ilegal, formal/ informal, riqueza/pobreza, inclusión/exclusión genera controversias y radicalidades está sujeta a instancias de poder, económicas y políticas, a la

¿Cuál es la meta propuesta? El objetivo es la superación, el progreso y el cumplimiento de metas sin romper las segmentariedades. conceptuales y, por ende, en los comportamientos sociales, en la economía y en la cultura. Al flexibilizar los conceptos relacionados con lo espacial y lo sociocultural, se propicia el cambio hacia una nueva conformación de ciudad ajustada a la contemporaneidad múltiple y diversa.

Según el filósofo Edgar Garavito (1999), "el desplazamiento de un concepto transforma la sensibilidad y los afectos del medio social que recibe el nuevo pensamiento" (p. 63) y los filósofos Deleuze y Parnet (1980) plantean que "la única forma de escapar de los dualismos es desplazarlos, hasta encontrar entre los términos un desfiladero estrecho semejante a una frontera, que va a convertir al conjunto en una multiplicidad independiente del número de partes" (p. 149). Sobre estos cuestionamientos se abre el intersticio y se construye la frontera, lo que facilita el desplazamiento conceptual y la inserción de lo "singular" como concepto que traslada la radicalidad y el modelo predeterminado y promueve un campo de acción que permite resignificar las condiciones socioespaciales como concepto constructivo de ciudad desde esta potencialidad.

Lo singular nace de la significación y pragmática desarrollada por Guattari y Rolnik (2005) como "una manera de rechazar todos esos modos de codificación prestablecidos, todos esos modos de manipulación y de control a distancia, rechazarlos para construir modos de sensibilidad, modos de relación con el otro, modos de producción, modos de creatividad que produzcan una subjetividad singular" (p. 28). Cuando el investigador
Estos rígidos paradigmas han dificultado la transición al entendimiento del pensamiento complejo contemporáneo, en el que se pone de manifiesto la dificultad en los desplazamientos 
se acerca a los barrios con la visión singular, se aleja de los códigos preexistentes y explora otras sensibilidades, otras maneras de relacionarse, de producir elementos diferenciadores potenciales para pensar ciudad.

Desde estas alternativas, la ciudad informal no puede ser vista desde su opuesto formal (Hernández García, 2011). Se vislumbra, entonces, la ciudad desde lo singular, para generar transformaciones y cambios trascendentales en su devenir, la ciudad con características y dinámicas propias independientes, razón por la cual las condiciones socioespaciales se movilizan y solucionan de forma diferente por su misma particularidad.

Lo singular deja entrever lo urbano desde la formas múltiples de construir ciudad y desde los tipos de desarrollo económico ajeno a las características del capitalismo corporativo y global, con prácticas sociales y culturales diversas a las consideradas homogéneas y hegemónicas, que abren paso a eso "otro" propuesto por el poblador desde su cotidianidad; el mapa mental que se construye es diferente, puede ser un plan "de composición”, como lo plantean Deleuze y Guattari (1997, p. 272), penetrado por el azar y el acontecimiento, de construcción de devenir.

Entonces, ¿cómo entender lo singular? ¿Cómo aprender a escuchar las voces de la diferencia y la diversidad social, a destacar el potencial creativo de la sociedad local? ¿Cómo reconocer las potencialidades y construir un territorio desde quienes lo habitan? ¿Cómo superar los discursos binarios para aproximarse desde otra perspectiva?

En este sentido, el artículo plantea el desarrollo del concepto de singularidad como un elemento que emerge desde las categorías opuestas y jerárquicas predeterminadas y propone una metodología denominada mapeo de lo singular, construida con líneas espaciales, socioculturales y económicas. Se desarrolla una exploración empírica en el sector de Suba-Tibabuyes (Bogotá) con resultados y cuestionamientos que invitan a desplazar el concepto de planificación urbana y de construcción socioespacial de la ciudad desde la fragmentación y la singularidad planteadas.

\section{En el intersticio de la oposición y de la jerarquización opera lo singular}

La construcción socioespacial de ciudad requiere un permanente cambio, originado desde la forma como habita el poblador y materializado en la transformación espacial. Intentar construir ciudad desde los imaginarios formales es desconocer que existen otras perspectivas. Es necesario contemplar las diferencias en las dinámicas que propicia la ciudad, no desde los opuestos ni desde las jerarquías, sino desde lo singular que surge por sí mismo.

Se entiende que la ciudad, como sistema, está compuesta por fragmentos con características diversas y singulares; no son partes homogéneas que se unen de acuerdo con lo similar, sino que se conectan por sus multiplicidades: "No se une desde lo homogéneo, se conecta desde lo heterogéneo" (Rojas, 2009, p. 223). No se puede seguir viendo la ciudad solo desde lo funcional o estructural; la complejidad dinámica hace tambalear el pensamiento lineal tradicional, porque la movilidad permite ser flexible en las categorías de periferia, pobreza, marginalidad y exclusión e inicia un cruce de fronteras e híbridos que imponen la indefinición, la mezcla y la superposición, con resultados instantáneos que, a su vez, son fragmentos en el tiempo, consistentes y contundentes para el momento en el que se requiere y sostenibles para un devenir que se construye.

Dentro de esta visión de superación de los discursos espaciales, sociales y académicos duales, el desarrollo del concepto de lo singular planteado 
en este artículo pretende prescindir de la discusión de las categorías de oposición formal/informal y poner en un campo en el que se acepte la multiplicidad de fuerzas y aspectos dinámicos que se movilizan en un territorio, en un fragmento de ciudad que posee unas características propias, que actúan por y en sí mismas. No solo trasciende el discurso de la hibridez al cuestionar los límites y las categorías puras para mezclarlas en la generación de una frontera, sino que va más allá, establece las relaciones y conexiones tanto de lo espacial como de lo económico, social (gestión, política) y cultural y deja ver todas las líneas que resquebrajan y emergen de la rigidez intrínseca de las categorías y jerarquías.

Godard dice: "No solo son importantes los dos campos que se oponen y se confrontan sobre la gran línea, la frontera también cuenta, frontera por donde todo pasa y huye siguiendo una línea quebrada molecular orientada de forma distinta" (citado en Deleuze y Parnet, 1980, p. 149). Más allá de las categorías que codifican y encierran al sujeto, al espacio y a lo económico en un deber ser, en un pertenecer, está aquello que emerge, que modifica, que se desvía, que resiste, que no es ni lo uno ni lo otro, pero que se hace presente para generar dinamismo y sucesos. Se ha producido un cambio en la distribución y en las relaciones: ha surgido algo nuevo.

Lo singular es lo que aparece, lo presente; es el aquí y el ahora; es tomar el elemento sin preconcepciones, categorías ni jerarquías; es lo existente, dejar hablar a ese otro, establecer la relación y construir, no desde la visión que se tiene de organización y planeación de un territorio, sino de todos los signos que emite sin que pase por la interpretación. Leer el signo, captarlo tal cual aparece y decodificarlo es el momento oportuno para iniciar la conexión de la diversidad singular que construye el plan de ciudad. Como plantea Calabrese (1999, p. 89), es el fragmento, por sí mismo, desde donde se puede establecer la conexión con otro; no es la parte de la ciudad la que tiene que acomodarse al esquema establecido y predeterminado, aun sin tener las características del modelo requerido.

Diferentes posiciones manifiestan el estado de transición contemporáneo de la oposición infor$\mathrm{mal} /$ formal y dejan entrever el desplazamiento conceptual necesario para plantear el cambio en la visión de ciudad, en algunos casos, sin romper la lucha de fuerzas entre los opuestos en la que lo formal se doblega ante lo informal o viceversa o en la que subsisten predeterminaciones. Kellett (2002) explica:

Los barrios informales [o populares] son ahora la forma dominante de producción urbana y de vivienda en las ciudades latinoamericanas. Estos asentamientos son la suma de acciones continuas de hogares de bajos ingresos, cada uno tratando de representar en términos físicos su visión de vida y valores a los que aspiran. Estas visiones son ambiciosas y requieren de una prodigiosa energía y creatividad, llevando a su vez a la aparición de una arquitectura doméstica híbrida rica en significado. La exploración de estos entornos auto-desarrollados puede ofrecer pistas acerca del importante rol de la arquitectura doméstica en la transformación y consolidación de las relaciones económicas y la identidad cultural (p. 28).

Es importante resaltar que para Kellett (2002) la arquitectura doméstica es la singularidad rica en significados que se abre paso en la frontera, desplaza la dicotomía y moviliza otros campos conceptuales, al dotarlos de significado, retroalimentarlos y definir características particulares.

Kellett (1995) también afirma que "lo formal y lo informal son sólo dos dimensiones que están intrínsecamente interconectadas: [...] la formalidad y la informalidad desde una variedad de disciplinas han demostrado que los dos sectores están lejos de ser independientes y separados" (p. 25). A pesar de que la investigación ha 
sugerido por años que lo formal y lo informal son caras de la misma moneda (Bromley, 1978; Kellett, 1995; Moser, 1994; Santos, 2000; Ward, 2004), todavía existe un dualismo vivo en el discurso alrededor de la ciudad formal e informal, tanto en la formulación de políticas como en el debate académico. Para algunos, la informalidad urbana "surge de un paradigma de liberalización" y no puede ser entendida fuera del contexto de la globalización como una vía alternativa de pensamiento y actuación: "La informalidad urbana no sólo es una alternativa económica y política, sino también una forma de vida" (AlSayyad, 2004, pp. 26-27). Cuando se consolidan estos asentamientos informales es imposible distinguirlos de los asentamientos iniciados formalmente; por una parte, los barrios informales tienden a "formalizarse" (Kellett, 2009), mientras los barrios formales tienden a "informalizarse" (Ceballos y Tarchópolus, 2003), con lo que se desdibuja la dicotomía formal/informal y sus implicaciones.

En esta transición conceptual también se reconoce que los asentamientos informales no solo comprenden una gran porción de las ciudades latinoamericanas, sino que también son una parte dinámica de estas en términos físicos, sociales y culturales. Fiori y Brandao (2010) argumentan que: "La informalidad urbana está entretejida inexorablemente con la ciudad como un todo - en todas las escalas y niveles- y tiene que ser vista como otra vía de ser en la ciudad y construirla" (p. 188). Los asentamientos informales son vistos, además, como innovadores y creativos: "Hoy reconocemos el genio innovador de los hogares de bajos ingresos, que toman ventaja de las oportunidades culturales específicas para sobrevivir y mejorar sus condiciones de vida" (AlSayyad, 1993, p. 5). Este reconocimiento del "otro" que constituye la totalidad de ciudad y de su potencialidad creativa contribuye a que se desvanezcan los discursos hegemónicos y su dominio de concepciones espaciales, económicas y socioculturales que invalidan la particularidad. Por lo contrario, se necesita estimular el respeto a la diversidad, a la alteridad como principio básico para pensar un territorio de convivencia ciudadana.

En términos de la espacialidad, los discursos que incluyen estos entornos están sujetos a distintas interpretaciones: nosotros no creemos que "informal" signifique in-forma (sin forma). Para nosotros implica que surge de sí mismo y de sus realizadores, cuya forma no ha sido reconocida aún, pero está sujeta a reglas y procedimientos potencialmente tan específicos y necesarios como aquellos que han gobernado la construcción de ciudad oficial y formal (Brillembourg y Klumpner, 2010, p. 120).

La diferencia se visibiliza en los espacios construidos cuando "el proceso de autoconstrucción realizado por el poblador surge sin planos ni ideas preconcebidas acerca de cómo organizar el espacio y el diseño es fruto de una práctica cotidiana de quienes lo habitan" (Skewes, 2005 citado en Zibechi, 2008, p. 68), en contraposición a las viviendas de interés social promovidas por la institucionalidad; en ellas, la propuesta de diseño y construcción son ajenas al poblador y son impuestas por otros que desconocen su cotidianidad. Con esto no se quiere plantear que solo la producción "informal" de espacio sea válida; todo lo contrario: la intervención pública debe ser más pertinente, informada y de acuerdo con las necesidades y circunstancias de los pobladores. Esto, entre otras cosas, es lo que la singularidad pretende aportar.

La singularidad emerge en el intersticio de cualquier oposición o jerarquización, en lo espacial urbano, en lo construido; surge en lo económico, en las prácticas sociales y culturales manifestadas y alejadas de la corriente principal. La expectativa y la sorpresa es su condicionante para atraparlas. 
Algunas prácticas sociales singulares encontradas en ciertas categorías pueden modificar estructuras sociales en pro de la convivencia, como las relaciones sociales arraigadas en valores de camaradería, reciprocidad, solidaridad y cooperación, lo que desestimula relaciones individualistas y competitivas, bases de sistemas sociales destructivos. Respecto a lo económico:

[...] se fortalece el desarrollo de estrategias de trabajo de supervivencia que buscan tanto la obtención de ganancias monetarias, como la creación de condiciones que favorecen el proceso de formación humana como la socialización del conocimiento, la cultura, la salud y la vivienda y, propician acciones colectivas organizadas en el ámbito de la comunidad. (Sarria y Tiriba, 2003, p. 3).

Lo singular económico se puede referir a un conjunto de prácticas en las que el poblador intenta asegurar a su modo la reproducción de la vida cotidiana, cómo produce y reproduce su existencia; no es necesariamente la comercialización del producto en algún lugar público, la falta de un vínculo de empleo, la relación empleador-empleado, el uso de la fuerza de trabajo como una mercancía o alguna forma de control por parte del Estado sobre las actividades económicas: se busca una esencia y singularidad en la concepción de vida económica resultante de la particularidad encontrada. Para Coraggio, esta puede ser "la lógica de la reproducción ampliada de la vida" (1997, citado por Sarria y Tiriba, 2003, p. 7). En estos términos, es preciso que se busque en la realidad concreta los datos empíricos que se refieran a todos los involucrados de una propuesta económica, para retroalimentar sus características y posibilidades como sistemas singulares y emergentes que movilizan los territorios.

Palma (1987) explica las actividades económicas en los barrios informales como "los valores, prácticas y expresiones culturales basadas en la ayuda mutua"(p. 32). Llama a estas prácticas "lo popular", que incluye participación de la familia y estrategias de supervivencia individuales y colectivas. "Lo popular" comprende también organización y cultura; para Palma (1987), "lo popular" y la pobreza no son lo mismo y lo popular no es definido por lo que carece, sino por las propuestas, iniciativas y contribuciones de las personas.

Estas [prácticas económicas informales] han pasado de ser observadas de transicionales a una formalización, a ser vistas como una alternativa válida y necesaria para la supervivencia de muchos en el continente. "Esta alternativa" no sólo comprende un tipo económico sino que es visto como un medio de organización que incluye dimensiones tanto sociales como culturales (Ruipérez Palmero, 2006, p. 105).

Así, "lo popular" puede verse como una categoría de "lo singular", que le añade significado a estas prácticas económicas y les da contexto para ser entendidas y, hasta cierto modo, valoradas.

Aunque todavía hay un dualismo vivo en el discurso alrededor de la existencia de una ciudad formal y otra informal, tanto en la formulación de políticas como en el debate académico, que incluye percepciones que han influido en la subvaloración de los asentamientos informales vistos como ilegales, marginales, fuente de problemas e incapaces de generar alternativas propositivas de cambio, algunas de las visiones actuales y alternativas sobre tales asentamientos buscan superar estos discursos binarios, aproximarse a ellos desde una perspectiva cualitativa y entenderlos dentro de su propio contexto, "enfatizando la naturaleza dinámica de los asentamientos informales constituidos como procesos sociales en lugar de categorizaciones estáticas" (Lombard, 2009, p. 296).

Como se expuso, algunos estudiosos del hábitat concluyen el respeto por las dinámicas espaciales y sociales que hay que tener para el estudio de estos asentamientos, reconocen que brindan una oportunidad de construcción de ciudad y constituyen un elemento primordial del espacio 
urbano; estas conclusiones se comparten en este artículo y se amplían desde la relación entre filosofía, contexto sociocultural que excluye o normaliza y urbanismo, para generar un punto de vista que aporte a esta controversia.

En apariencia, la discusión del planteamiento se desarrolla en el campo filosófico y se reconoce como la esencia en el constructo del discurso; a partir de esclarecer y aprehender el concepto de lo singular sucede el desplazamiento del pensamiento, sale de las preconcepciones, muestra otro paradigma que asume la complejidad desde la multiplicidad y acerca al conocimiento de algo que se desconoce desde otro concepto sin categorías, sin jerarquías, sin predeterminaciones ni interpretaciones; solo conocer desde lo que surge. Esta esencia permite trascender las fronteras de la oposición para descubrir las conexiones que nacen de las diferentes fuerzas que se cruzan en el territorio. Lo singular busca esta interconexión y aportar además una comprensión socioespacial más clara y emergente de ciudad.

La aplicación o la pragmática del concepto de singularidad, según Guattari y Rolnik (2005), saca de la oposición y jerarquización el pensamiento moderno occidental atrapado en estas categorías e inicia el desplazamiento o la transformación de un saber acorde con la complejidad contemporánea, que promueve otra forma de pensar sobre los cuestionamientos urbanos y sociales. Es un momento histórico complejo que requiere otras herramientas y otros conceptos; la creación de nuevos sentidos.

¿Cómo descubrir la singularidad barrial en los intersticios de las oposiciones? ¿Cómo desplazar estas dicotomías y construir conceptos que transformen el medio y el pensamiento social? ¿Cómo buscar en la realidad concreta los datos empíricos que se refieran a los autores e interlocutores de propuestas económicas, sociales, espaciales y culturales singulares?

\section{Metodología}

En la exploración de las preguntas anteriores se construye un planteamiento metodológico que parte del concepto de "plan de composición” desarrollado por Deleuze y Guattari (1997, p. 272), el cual contempla la composición de un trazado que viene de la especificidad y singularidad de todo campo de acción, no diseñado con un fin determinado y con anterioridad, sino que recurre a conocimientos prácticos y emergentes del territorio en el momento actual. Se refiere, entonces, a "la puesta al día de un trazar que no viene guiado por ninguna intención” (Álvarez de Toledo, 2009, p. 8); este trazado tiende a la búsqueda de la complejidad y singularidad territorial y a acercarse para descubrir los movimientos, aquellos difíciles de discernir que fluyen en el intersticio, las invenciones entre segmentariedades; lo que transita por el medio y propone otras cartografías en diferentes campos: económico, espacial, social, cultural y político.

Como estrategia metodológica, se plantea el mapeo de lo singular, que consiste en el levantamiento de sucesos reales, transitorios y dinámicos en un territorio donde constantemente aparecen modificaciones. Este mapeo se construye por medio de todas las líneas sensibles, emergentes, funcionales, jerárquicas, que surgen o se encuentran estáticas. Puede aparecer lo habitual, lo cotidiano, lo extrańo, lo que está demasiado atrapado en las categorías, los afectos, la institución (Estado, Iglesia, familia).

Las líneas del mapeo se construyen desde los trayectos, desde el trazado de rutas a partir de lo inesperado y de líneas con trazos no predeterminados; se abre paso al azar, a la posibilidad de encuentro con lo otro, a la inestabilidad e inseguridad; siempre se está a la expectativa de que algo surja. La información es constituida por elementos aún no formados. Se entienden las 
relaciones de movimiento, dinamismo o reposo del territorio y converge todo tipo de fuerzas. Se pretende reunir lo heterogéneo, lo heteróclito (singular, extraño) y asegurar la consolidación de las multiplicidades.

Es importante reconocer que se opone a todo plan de principio o de finalidad e implica evitar categorizar, jerarquizar y significar, abstraerse de la totalidad dada para indicar una resignificación que parta de las singularidades de lo existente. Es necesario estar alerta para leer y atrapar aquello que emerge y fluye para armar cartografías. Las fuerzas o "líneas del errar", como lo plantea Fernand Deligny (1971), crean una cartografía que transcribe los trayectos en el espacio y manifiesta el actuar "para nada" y lo "humano por naturaleza”. Los trayectos contribuyen de manera significativa a establecer la singularidad, desde donde surgen otras organizaciones socioespaciales, culturales y económicas que posibilitan un entendimiento "desde el lugar" y "para el lugar".

Los métodos usados para construir el mapeo de lo singular aparecen de la manera como se aproxima el investigador a lo que quiere conocer; en este caso, al territorio. Plantea el andar como derivar, errar o acción que constituye los recorridos de exploración, de reconocimiento, sin puntos de referencia estables, espacios ni lugares predeterminados, a la deriva, donde se tiene la opción y la capacidad para construir, a cada instante, un propio mapa e indagar sensorial y perceptualmente un territorio (Rojas y Osorio, 2011, pp. 37-38). Francesco Careri (2002, p. 186) y su grupo Stalker (investigadores dedicados al arte urbano, con especial atención en las zonas periféricas de las ciudades) implementaron la "transurbancia", que se trata de pasear, de recorrer el territorio y levantar mapas no convencionales, de recuperar el descubrimiento de la ciudad llena de contradicciones. La observación directa como medio para indagar y descubrir lo incomprensible, aquello que se escapa a las categorías tradicionales (formal/informal), para leer signos, ver y trazar sin interpretar, según Deligny (1971) y, como dice Joan Costa (2003, citado en Gómez y Londońo, 2011, p. 38) “visualizar' para ampliar el mundo de lo perceptible, desde donde se generan y transmiten conocimientos que al principio no están a nuestro alcance, donde se encuentran nuevas dimensiones geofísicas, nuevas relaciones con los lugares". Las conversaciones no estructuradas con los pobladores, en la medida en que se va andando, constituyen un medio importante para entender y dar sentido a situaciones o temas no apreciables a simple vista o que necesitan información cualificada por parte del poblador para comprender las lógicas internas. Para apoyar todo el proceso se cuenta con la fotografía, que registra lo que sucede, permite construir la integralidad de un suceso y conectar fragmentos de vida, de trazos, afectos y pasiones que surgen cuando se observa y se frecuentan ciertos lugares. "La fotografía provee de una clase de información que es difícil de representar con palabras" (Wagner, 2007, p. 4). La fotografía como intermediaria, como contacto sensible a lo invisible, representa la ausencia emocional que produce la velocidad de los informativos audiovisuales, atrapa las sensaciones de tristeza, soledad, hacinamiento y cansancio del errante que aparece en el escenario urbano.

Todo lo anterior se registra en un diario de campo, como información que contribuye a la construcción de mapas y narrativas que nos aproximan a la singularidad. Cada salida, ruta, observación y conversación queda registrada y se convierte en un punto que deriva en varios, retroalimenta el mapa y muestra el camino por donde hay que seguir. Estos intersticios están movilizados por el mapeo de lo singular por medio de una "narrativa cartográfica" que da cuenta de potencialidades y carencias, de historia e ilusiones y de generalidades y particularidades que permiten entender las dinámicas socioespaciales del lugar desde otra perspectiva 
y alternativa a la planeación tradicional, lo que hace visibles a los actores como ejes fundamentales de su desarrollo.

Se inicia al acercarse al territorio, errar por él y explorarlo, sin finalidad ni objetivo. Con un mapa de localización del territorio, se realiza una georreferenciación de los trazos por donde se va andando y sobre este mapa se van resaltando las particularidades encontradas, aquello que llama la atención o es diferente a los elementos comunes; luego se hace un geoanálisis que integre estas características al levantamiento realizado, lo que da como resultado una confrontación del espacio físico actual con el fotografiado digitalmente y sobre el cual se inserta lo encontrado. Paralelo al andar, se observa la manera como habita la población, como usa el espacio y se registra en un diario de campo, mediante dibujos, señalamientos, bocetos de las vías, anotaciones sobre las conversaciones de lo que sucede en los espacios singulares y fotografías. El diario es la herramienta fundamental para el posterior análisis de la información y el punto de partida para continuar con la otra ruta del mapa: la caracterización social del territorio. Las narrativas socioespaciales marcan los cambios singulares que deben tenerse en cuenta al momento de planear un territorio: el uso, la manera como acceden y se apropian del territorio, la facilidad y el manejo de circulación y su distribución espacial de acuerdo con la labor desempeñada.

Desde estos planteamientos metodológicos se analiza un sector popular de la ciudad de Bogotá, para explorar lo singular de su ocupación socioespacial y para lograr un entendimiento desde los mismos actores y desde el mismo lugar, que brinde herramientas no solo para su comprensión, sino también para su planeación y desarrollo. Tales herramientas son quizá distintas a la visión cuantitativa, de arriba abajo y solo de consideraciones económicas o de mercado, del diagnóstico, de la planeación y del desarrollo urbano tradicional.

El caso estudiado es el sector de Suba-Tibabuyes, ubicado en la localidad 11 (Suba) de las veinte en las que está dividida la ciudad, en el noroccidente de Bogotá. De este sector se caminaron los barrios de Santa Cecilia I y II, Villa Cindy, Santa Rita y Lisboa por su borde, que es el río Bogotá. El estudio se focalizó en las comunidades de recicladores ${ }^{1}$ asentadas en estos barrios, que son las menos escuchadas $y$, en gran medida, las más invisibilizadas del sector.

Los resultados que se presentan a continuación forman parte de una investigación de un colectivo social independiente, conformado por los investigadores y apoyado por un promotor social, Wilson Cárdenas y por quienes en ese entonces eran estudiantes (hoy profesionales) de Arquitectura, José Ignacio Torres y de Licenciatura en Artes Plásticas, Camila Cifuentes. La investigación se llevó a cabo entre marzo de 2011 y julio de 2012, con salidas de campo irregulares, aleatorias y dependientes del tiempo que durara el análisis de la información recogida, la retroalimentación y la alternativa de dirección de búsqueda que se propusiera.

El mapeo de la singularidad del sector se construyó a partir de las múltiples salidas de campo que originaron, por una parte, un mapeo del ordenamiento espacial del territorio de acuerdo con las dinámicas de uso y apropiación del poblador $y$, por otra parte, un mapeo sociocultural que surge de las características laborales y culturales de una población específica. Por último, un mapeo económico que potencia la comercialización del material y el mercado del sector y de la ciudad. A continuación se señalan los principales hallazgos y conexiones. 


\section{Lo singular, el caso de Suba-Tibabuyes}

La aplicación del mapeo de lo singular permitió entrever el fragmento por sí mismo, sus singularidades y la conformación de la población recicladora, que impone su dinámica social y de vivienda en un territorio de apropiación en donde vive su cotidianidad y genera hábitos y costumbres de acuerdo con su actividad laboral, sus recursos y deseos. Esta población se encarga de proponer una cartografía de resignificación de adentro hacia afuera. Los trazos en el territorio se van evidenciando con los desplazamientos, las rutas por donde se anduvo y el registro de las particularidades. En la figura 1 se muestran algunas características de la cotidianidad de los recicladores relacionadas con movilidad, vivienda, lugares de recolección y algunas posibilidades de organización territorial singular.

En tanto el andar se fue desarrollando, se evidenció la existencia de una división en el uso del territorio referido a la labor del reciclaje, otro resultado singular territorial para tener en cuenta en una planeación. Mientras en los barrios de Santa Rita y Villa Cindy se realiza en mayor medida la selección y clasificación del material, en los barrios Lisboa y Santa Cecilia se almacena, distribuye y comercializa. En estos barrios se encuentra el mayor número de centros de acopio (lugar donde se almacena y comercializa el material reciclable). Además, se encontró que en la

Figura 1.

Características y posibilidades generadas en el territorio de la población recicladora

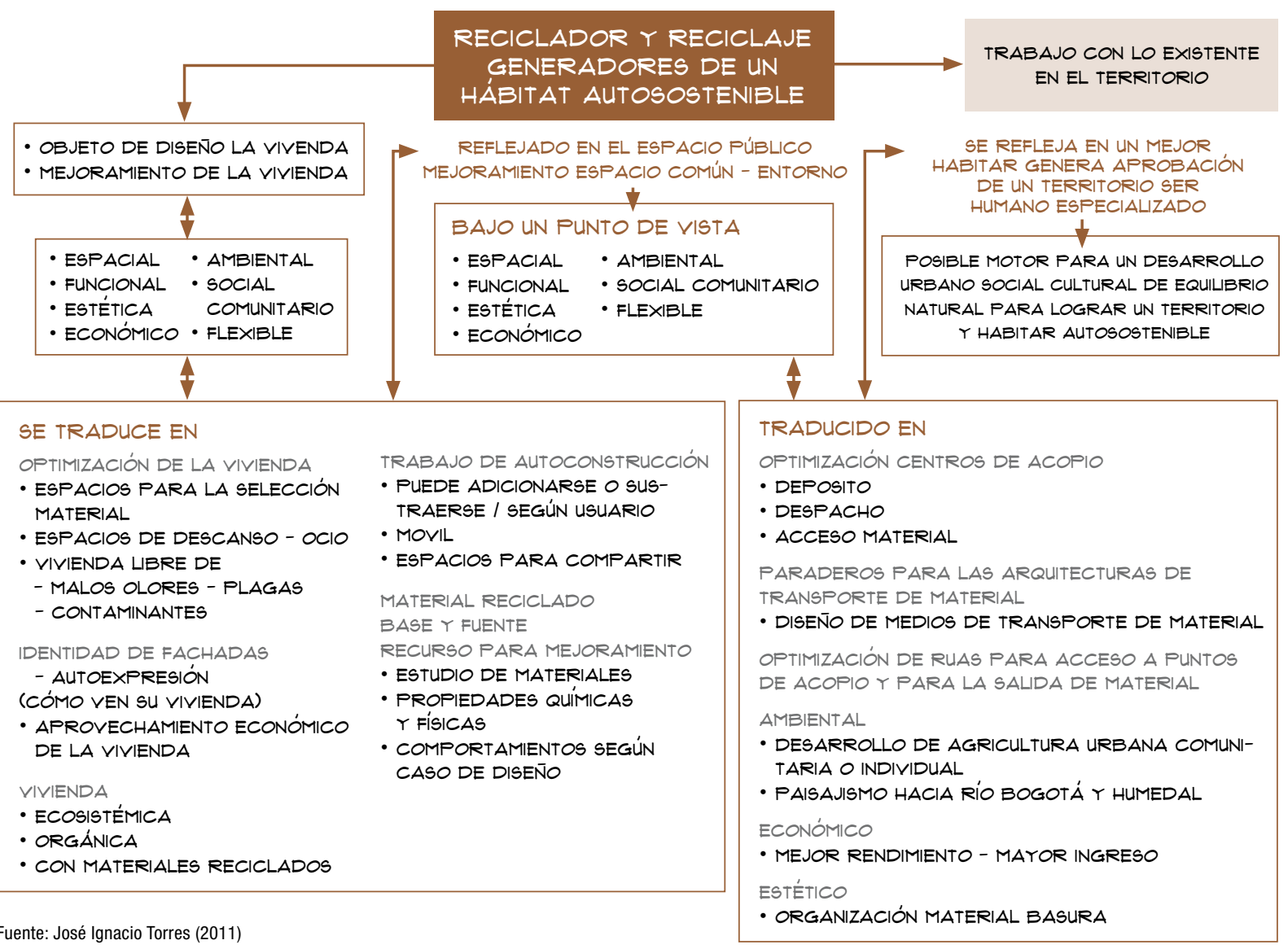


ronda del humedal Juan Amarillo también se almacena y existe la venta de flejes (hierro doblado para construcción). Si para efectos de una planeación urbana se fuera a considerar esta lectura, el sector se distribuiría de acuerdo con esta especificidad de la cadena de reciclaje en pro de "usos del suelo". La figura 2 muestra la georreferenciación de lugares y nodos donde convergen actividades, encuentros sociales, flujos, fuerzas y economías de los recicladores.

Errar, derivar, observar, fotografiar, recoger y plasmar la información se convierte en el proceso metodológico en la construcción del mapeo de lo singular. La figura 3 muestra la localización de los veintiocho centros de acopio encontrados, entre los cuales están veinte bodegas de almacenamiento y clasificación al aire libre, en donde se selecciona y almacena material; ocho bodegas de comercialización caracterizadas por la compra y venta de material y catorce bodegas de flejes dedicados al almacenaje y doblaje de la varilla de hierro. ¿Cómo hacer una planeación territorial que tenga en cuenta la ubicación, el funcionamiento y el aporte que hacen al hábitat y al entorno estos centros? ¿Los desalojaríamos del lugar o los incorporaríamos a los planes zonales? ¿Cómo involucrar a estos pobladores a las dinámicas de reciclaje en la ciudad? Surgen muchas preguntas más que permiten movilizarnos de la planeación urbana predeterminada a otro tipo de composición originada por una dinámica territorial del poblador.

En uno de los trayectos, los pobladores nos hicieron ver que la vivienda "es parte del espacio urbano" (o el espacio urbano es parte de la vivienda) por su estrecha relación espacial y productiva, es decir, la actividad del reciclaje se extiende a la calle y de la calle se extiende a la vivienda. El acercamiento a la vivienda permitió observar que algunos recicladores construyen espacios de bodegaje o vivienda con el material que consiguen y conforman un collage de materiales y de técnicas, la mayoría de las veces organizados
Figura 2.

Georreferenciación de las actividades realizadas por los recicladores sobre la ronda del río

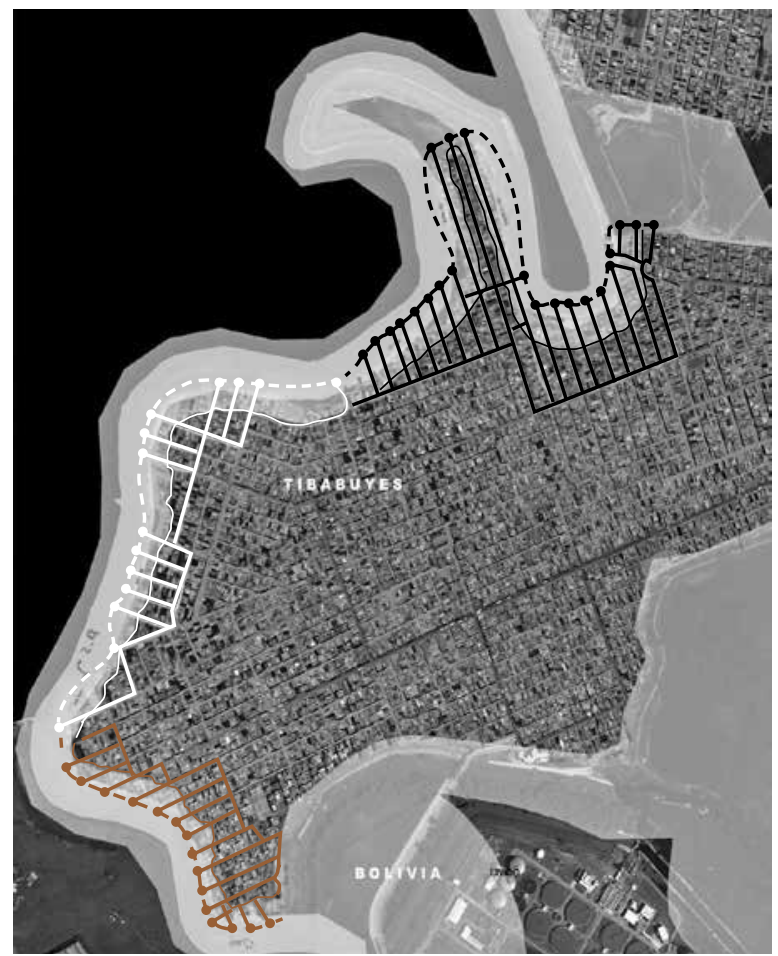

Fuente: José Ignacio Torres (2011)

En café, siembra de pancoger en el barrio Santa Cecilia I; en blanco, clasificación de material reciclable en los barrios Santa Cecilia II y Villa Cindy; en negro, recreación y ocio en el barrio Santa Rita.

por adición o superposición de capas. Cuando la vivienda es propia, se va desarrollando y ampliando de acuerdo con las necesidades del proceso de autoconstrucción; cuando es alquilada, un espacio de ella está dedicado a almacenar el material y a guardar su medio de transporte (bicicleta, zorro o carruaje). El reciclador percibe el espacio abierto como la prolongación del trabajo del reciclaje: se apropia de los lotes vacíos, de la ronda del río, del humedal y de las calles (figuras 4 y 5). La calle es el espacio de mayor apropiación, pues se inunda de costales de gran tamaño (globos) cuando la actividad es la selección del material y el empaque o es lugar de parqueo de zorras (carros de tracción animal), carretillas, camiones y globos, de alimentación para el caballo, de carga, de juego, de charla. ¿Cómo reflejar estos aspectos en un diseño arquitectónico 
Figura 3

Elementos del mapa singular del reciclaje en Suba-Tibabuyes

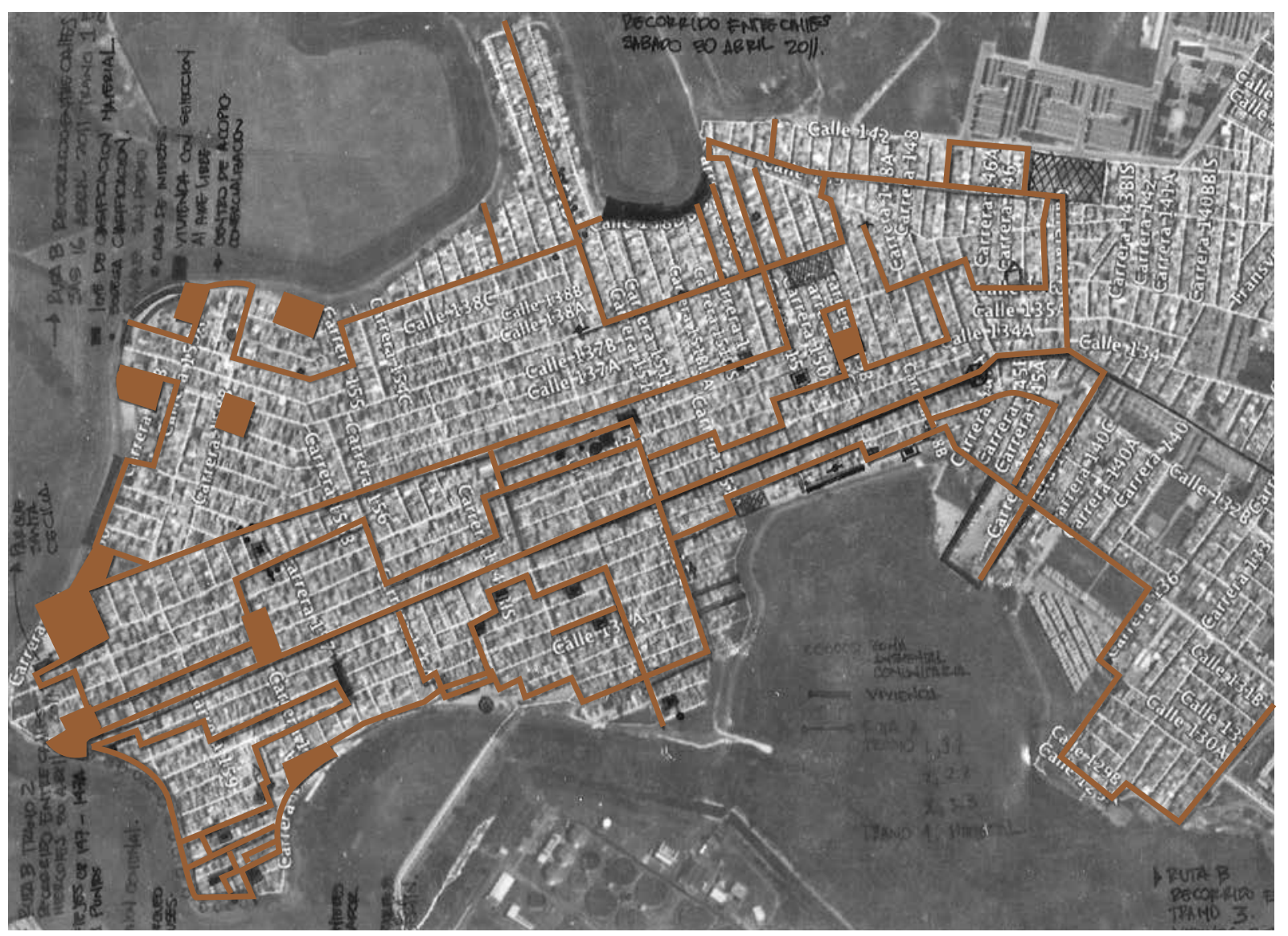

Fuente: José Ignacio Torres (2011)

Figura 4.

Prolongación de la actividad del reciclaje, de la vivienda al espacio abierto urbano

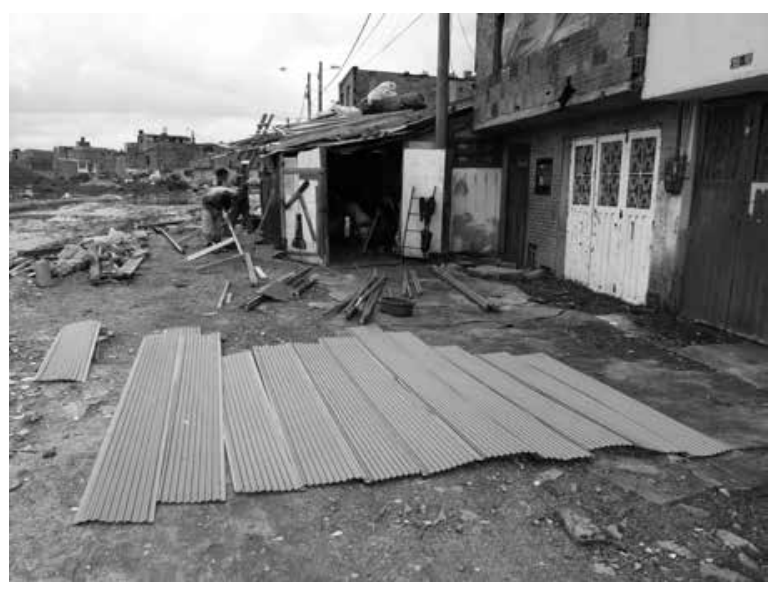

Fuente: Darío A. Jiménez (2011)
Figura 5.

Almacenamiento de material reciclable en el espacio abierto urbano

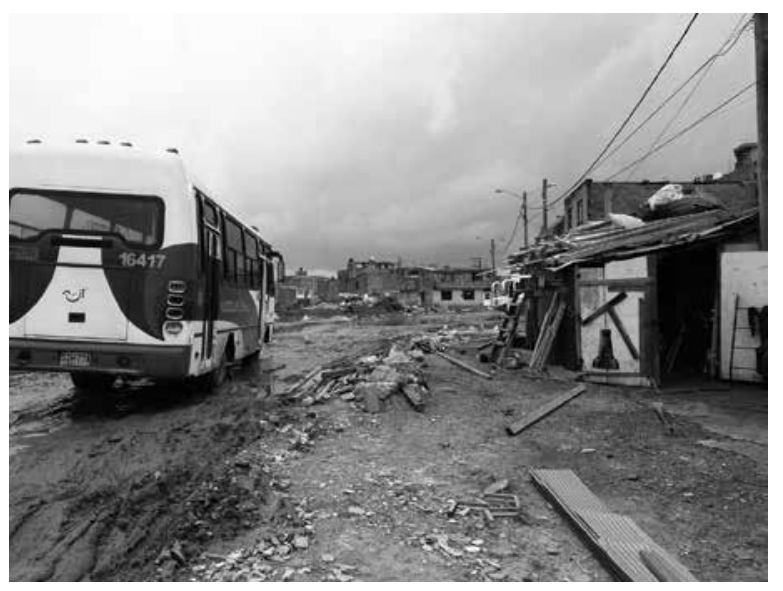

Fuente: Darío A. Jiménez (2011) 
formal? ¿Cómo los planes de vivienda de interés social (VIS) o prioritaria (VIP) del distrito capital asumen estas características de espacialidad? ¿Cómo integrar la relación público-privada en el desarrollo espacial tanto de la vivienda, como del espacio público? La evidencia de los desarrollos formales de vivienda desconocen la espacialidad que requiere el reciclador, la forma de economía y apropiación de su hábitat y lo que se encuentra en el mercado son soluciones carentes, que no cumplen las expectativas y necesidades de las personas y además genera un endeudamiento que va en contra de sus posibilidades.

Pensar en diseñar una calle en el sector se desdibuja de los parámetros convencionales. Se debe analizar y vincular otros requerimientos. La jerarquía vial de principal, secundaria y terciaria desaparece y la traza urbana que hace el reciclador evidencia un uso distinto del trazado ¿Cuáles son las calles que usan para seleccionar el material? ¿Cuáles para parqueo? ¿Cuáles se encuentran o cierran? Hay diversidad en diseño, circulación, peatonalización, ciclorrutas, etc. Vemos que estos resultados propician la transformación espacial desde otros requerimientos a tener en cuenta tanto en la vivienda como en el espacio público (cambio de diseño de andenes, de calles, de bahías de parqueo) y a priorizar vías y su flujo o circulación.

El mapeo del ordenamiento espacial del territorio muestra que la manera como transitan los recicladores sugiere intervenir una serie de vías locales prioritarias para ellos e iniciar la apertura y la permeabilidad de la periferia a los centros de la ciudad, para incluir el fragmento y conectarlo física y socialmente. Esta apertura estaría basada en la población del sector, sus recorridos principales, flujos, formas de comunicarse y espacios que une. El desarrollo económico sostenible del sector promueve el desplazamiento de la inversión y costos a la generación de una infraestructura viable y de fortalecimiento.
El mapeo económico es la actividad del reciclaje - esencia misma de la ocupación del territorio-y se vincula con lo físico-espacial y lo sociocultural. La exploración de lo singular en lo económico es complementada con conversaciones sostenidas con los recicladores del sector respecto a las rutas por donde recogen los residuos sólidos, el tipo de material que reciben y su comercialización, con preguntas como ¿Cuáles son los materiales que más recogen y comercializan en el barrio? ¿Papel, cartón, metal, flejes, plástico? ¿Cómo lo distribuyen? ¿Por dónde sale del sector y hacia dónde lo distribuyen? ¿Conocen industrias de pretransformación y transformación de material (concentrados, plástico)? Las respuestas marcan trazos posibles para adecuar el espacio a este tipo de uso (figuras 6 y 7 ) y permiten establecer un plan de negocios que surja de la forma de operar en el territorio. ¿Cómo manejan los precios de compra y venta del material, que por lo general son respetados por todos los centros de acopio y comercialización del lugar? ¿Cuáles son las características del mercado en lo relacionado con el comercio internacional? Conocen muy bien los meses cuando baja la comercialización y los meses cuando aumenta, así como los momentos en que las empresas de plásticos recogen el material. A partir de estos datos se puede pensar en una red empresarial de negocios, de organizaciones sociales solidarias que sean sostenibles y no tiendan a la desaparición.

El mapeo sociocultural se construye fundamentalmente por la voz del reciclador, del poblador, de la institución, del desplazado, del joven y del arrendatario, lo que da cabida a escuchar diferentes puntos de vista que manifiesten la diferencia. Esta narrativa, surgida de la interacción, elabora una cartografía social desde la multiplicidad de discursos, de posiciones, de opiniones que forman un territorio social y cultural específico. Por ejemplo, un discurso que se cruzó con la información obtenida en el territorio fue el institucional, al tener en cuenta los decretos y las normas vigentes 
relacionadas con el reciclaje, con las propuestas de vivienda de interés social o de interés prioritario, con el Plan de Ordenamiento Territorial (POT) y con los planes de desarrollo y organización con objetivos predeterminados para esta población.

Durante la construcción de los mapas, se encontró que los recicladores también estaban trazando su discurso de manera autónoma, con el interés de vincular a los otros pobladores del sector para sensibilizarlos frente a su quehacer, iniciar una integración social, establecer una estrategia de acercamiento al modo en que realizan su trabajo y contribuir en la construcción de un territorio sostenible. Estaban promocionando diversas actividades, como talleres de formación destinados al reconocimiento y manejo del material reciclable con cartón y PET (poliestireno), materiales que, transformados, permiten ser revertidos en objetos utilitarios, en la construcción de la vivienda y cambios en el entorno. Asimismo, se promovía la movilización de los pobladores en la apropiación de espacios abiertos, mediante la propuesta de unas "tomas transitorias e itinerantes" para compartir en comunidad, realizar trabajos con el material reciclable y jugar con los niños. Estas tomas se llevaron a cabo en la ronda del río

Figura 6.

Apropiación de la ronda de rio por parte de los recicladores

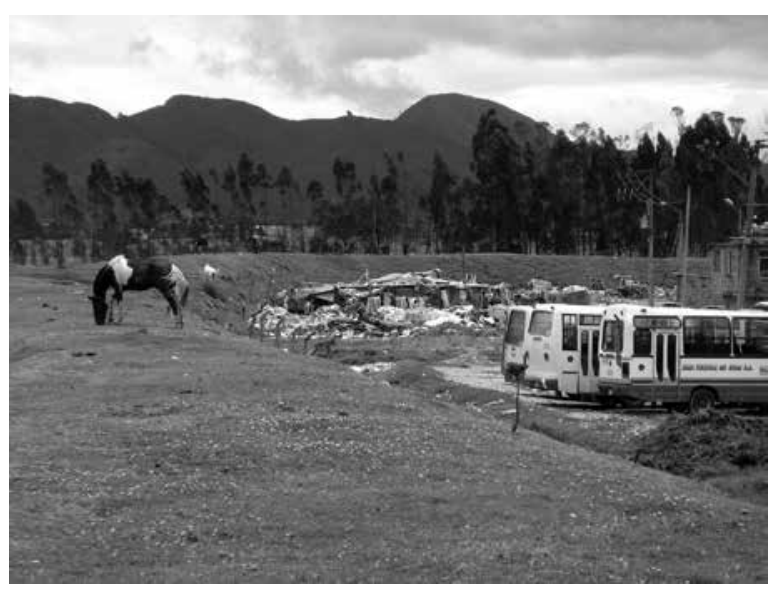

Fuente: Camila Cifuentes (2012)
Bogotá en un sector denominado La Bota, el parque de Villa Cindy y Berlín (figura 8).

Todas estas particularidades y su conexión fundamentan y construyen el mapeo de lo singular. Tener en cuenta los resultados en cada uno de los territorios que se exploran y la composición de cambio resultante sería ideal para la transformación socioespacial; la generación de alternativas que surgen en el intersticio muestran otra opción de planeación urbana desde otra percepción de un fragmento de ciudad que permite ver lo que fluye en el medio y los movimientos particulares.

La identificación de lo singular y su materialización en cartografías y narrativas pueden incitar a los investigadores y a los planificadores urbanos a cuestionarse sobre lo que consideran que es el orden o la organización de un territorio y puedan a partir de otras perspectivas, generar innovación en la planificación y contribuir a la creación de nuevos paradigmas. El acercamiento a lo desconocido con el interés de buscar en cada instante la esencia cualitativa y dejar de lado las asociaciones y los modelos preexistentes posibilita que aflore en los espacios intersticiales eso que se desconoce, que emerge singular, que no obedece a

Figura 7.

Centros de acopio locales y su organización por materiales

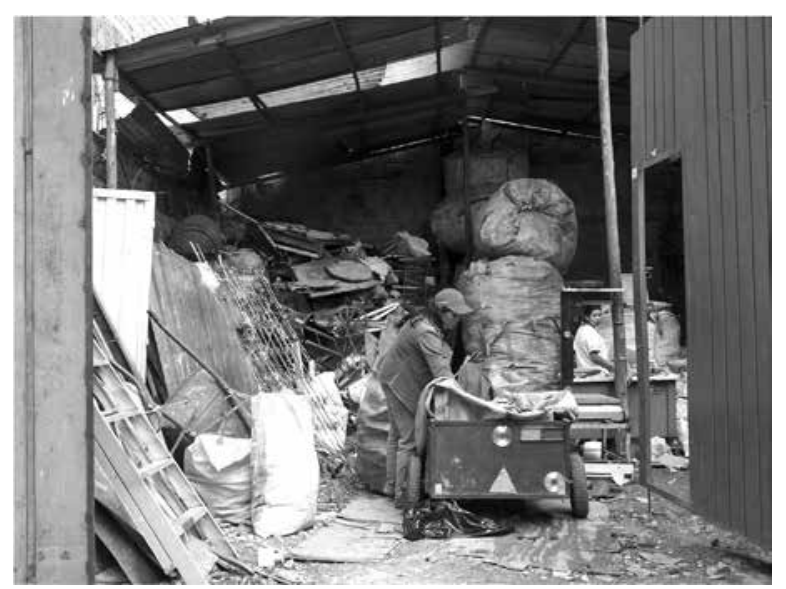

Fuente: Camila Cifuentes (2012) 
Figura 8

Tomas transitorias e itinerantes realizadas por los recicladores en la ronda del río Bogotá

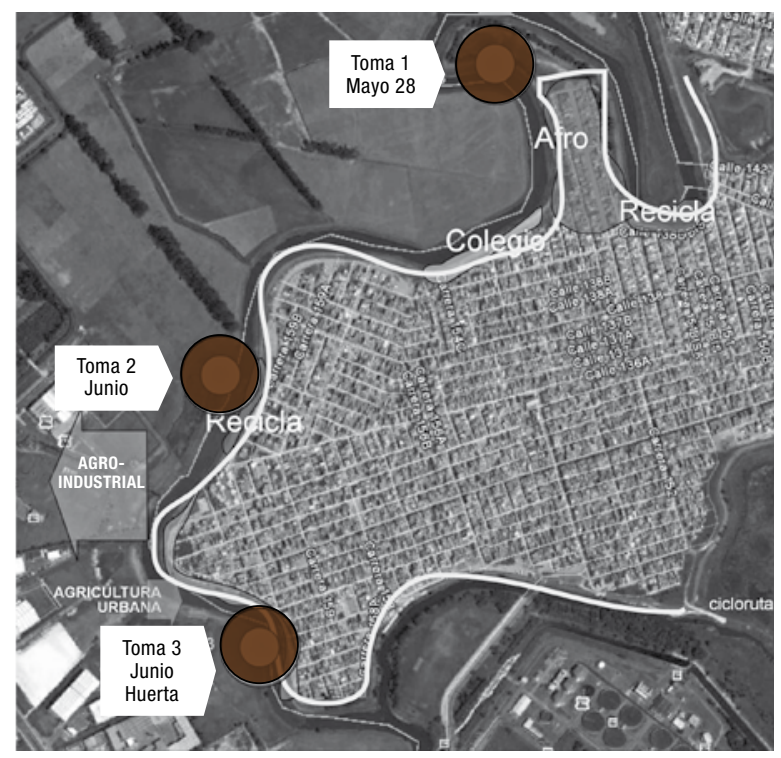

Fuente: Henry Osorio (2012)

parámetros espaciales, sociales, económicos, culturales genéricos o universales; es permitir que ingresen las diferentes líneas a un campo de acción que, en este caso, es el territorio, que se crucen y conecten los diferentes discursos que lo dinamizan, que las variables temporales se movilicen en el momento oportuno y que fluyan, sin presión del tiempo condicionado por la funcionalidad y la velocidad.

Concretar la ejecución de las resultantes de estos mapas contribuye a la conformación de la ciudad fragmentaria singular $y$, en consecuencia, a que las condiciones sociales de apropiación por parte del poblador de su hábitat estén acordes y coherentes con lo que ellos mismos han creado y aprehendido.

\section{Conclusiones}

Se considera que lo singular es una alternativa que ayuda a disolver las dicotomías férreas (formal/informal, legal/ilegal, pobreza/riqueza) desde donde se han visto la ciudad, la economía y la sociedad para tener otra visión de ciudad, de pobreza y de mejoramiento urbano. Fue importante encontrar un concepto que desplazara esta dicotomía, borrara el límite entre los opuestos y estableciera la frontera que dejara entrever la multiplicidad.

Lo singular puede ayudar a comprender mejor los llamados asentamientos informales o populares, los cuales son una realidad evidente en Colombia y en América Latina y han sido, para buena parte de la población, la única forma de acceder a vivienda y servicios urbanos. A pesar de sus carencias y problemáticas evidentes, son el presente para una gran porción de la población urbana en la región, que pueden dar lecciones importantes en términos de riqueza formal, ingenio individual y colectivo (Brillembourg y Klumpner, 2005); además, ofrecen una perspectiva para entender diferentes formas de ver y pensar la ciudad (Brillembourg, Feireiss y Klumpner, 2005; Hernández y Kellett, 2010; Roy, 2009). La identificación de lo singular y su espacialización no solo física, sino también social, económica y cultural van en el mismo sentido. No se trata, entonces, de una visión romántica del tema: se trata de construir territorio a partir de las propias posibilidades del mismo y desde la voz de sus pobladores.

El barrio se trata como un fragmento singular que da la alternativa de iniciar una conexión con otros no desde la totalidad de ciudad homogénea, en donde cada espacio es programado desde la norma existente así no tenga las características para que surja ese cambio o desarrollo, sino desde la heterogeneidad que relaciona y conecta. El fragmento permite realizar una intervención puntual y eficaz, con la intención de transformar la ciudad, fragmento a fragmento. Al ser intervenido, se inicia una resignificación de los lugares tradicionales, mediante una relectura de 
los elementos urbanos para lograr importantes efectos de recuperación urbana en un entorno próximo. Entonces, no tenemos barrios informales, pobres, periféricos, ilegales, sino barrios construidos desde sus potencialidades diversas, múltiples, con dinámicas económicas sostenibles.

Esta transformación de pensamiento puede producir un proyecto urbano de planeación y organización participativa que implica el levantamiento de las condiciones actuales de los lugares, de singularidades y heterogeneidades sociales, espaciales (viales y de espacio público) y económicas, como componentes trazados por los pobladores cuya propuesta genera otra organización espacial. Un método inductivo que construye devenir.

Es difícil construir ciudad sin modelos o parámetros predeterminados, desde las particularidades; el plan lo brinda lo existente, el dinamismo propio del sistema. ¿Cómo hacerlo? ¿Cómo aprender a escuchar al otro? ¿Cómo atrapar lo desconocido? ¿Cómo dejar de lado la categoría "pobreza" y sus denotaciones e imponer la potencialidad? Este es el reto: aprender a construir territorio de manera distinta.

\section{Referencias}

AlSayyad, N. (1993). Informal Housing in a Comparative Perspective. On Squatting, Culture, and Development in a Latin American and a Middle Eastern Context. Review of Urban and Regional Development Studies (5), 4-15.

AlSayyad, N. (2004). Urban Informality as a "New" Way of Life. En A. Roy y N. AlSayyad. Urban Informality: Transnational Perspectives from the Middle East, Latin America, and South Asia (pp. 7-30). Nueva York: Lexington Books.

Álvarez de Toledo, S. (2009). Fernand Deligny. Permitir, trazar, ver. Barcelona: Museo de Arte Contemporáneo de Barcelona (Macba).
Brillembourg, A. y Klumpner, H. (2010). Roles of Engagement: Caracas and the Informal City. En F. Hernández, P. Kellett y L. Allen (eds.), Rethinking the Informal City: Critical Perspectives from Latin America (pp. 119-136). Oxford: Berghahn Books.

Brillembourg, A., Feireiss, K. y Klumpner, H. (eds.) (2005). Informal City: Caracas Case. Munich: Prestel.

Bromley, R. (1978). The Urban Informal Sector: Why is it Worth Discussing? World Development, 6(9-10), 1033-1039.

Calabrese, O. (1999). La era neobarroca. Detalle y fragmento. Madrid: Cátedra.

Careri, F. (2002). Walkscapes, el andar como práctica estética. Barcelona: Gustavo Gili.

Ceballos, O. y Tarchópulos, D. (2003). Calidad de la vivienda de sectores de bajos ingresos. Bogotá: Centro Editorial Javeriano.

Deleuze, G. (1995). Prousty los signos. (F. Monge, trad.). Barcelona: Anagrama. (Original publicado en 1964).

Deleuze, G. y Guattari, F. (1997). Mil mesetas. Capitalismo y esquizofrenia. Devenir-intenso, devenir-animal, devenir-imperceptible. Madrid: Pretextos.

Deleuze, G. y Parnet, C. (1980). Diálogos. Valencia: Pretextos.

Fiori, J. y Brandao, Z. (2010). Spatial Strategies and Urban Policy: Urbanism and Poverty Reduction in the Favelas of Rio de Janeiro. En F. Hernández, P. Kellett y L. Allen (eds.), Rethinking the Informal City: Critical Perspectives from Latin America (pp. 181-205). Oxford: Berghahn Books.

Garavito, E. (1999). Escritos escogidos. Medellín: Universidad Nacional de Colombia. 
Gómez, A. y Londoño, F. (2011). Paisajes y nuevos territorios (en red). Barcelona: Anthropos.

Guattari, F. y Rolnik, S. (2005). Micropolítica. Cartografias del deseo. Madrid: Traficantes de sueños.

Hernández García, J. (2011). Espacios públicos en barrios informales: producción y uso entre lo publico y lo privado. Mexico: Infonavit y Redalyc.

Hernández, F. y Kellett, P. (2010). Re-Imagining the Informal Settlements in Latin America. En F. Hernández, P. Kellett y L. Allen (eds.), Rethinking the Informal City: Critical Perspectives from Latin America (pp. 1-19). Oxford: Berghahn Books.

Kellett, P. (1995). Constructing Home: The Production and Consumption of Popular Housing in Northern Colombia. (Tesis doctoral inédita). University of Newcastle, Newcastle upon Tyne, Reino Unido.

Kellett, P. (2002). The Construction of Home in the Informal City. Journal of Romance Studies, 2(3), 17-31.

Kellett, P. (5 de abril de 2009). Original copies? Imitative Housing Forms and Dwelling Practices in a Squatter Settlement. Ponencia presentada en Anthropological and Archaeological Imaginations: Past, Present and Future. Bristol, University of Bristol.

Lombard, M. (2009). Making Place in the City: Place-Making in Urban Informal Settlements in Mexico. (Tesis doctoral inédita). University of Sheffield, Sheffield, Inglaterra.

Moser, C. (1994). The Informal Sector Debate, Part 1: 1970-1983. En C. Rakowski. Contrapunto: The Informal Sector Debate in Latin America (pp. 11-30). Albany: University of New York Press.

Osorio, H. y Rojas, E. (2011). La cartografía como medio investigativo y pedagógico. dearq (9), 30-47.
Palma, D. (1987). La informalidad, lo populary el cambio social. Lima: Desco.

Rojas Sánchez, E. (2009). Negación de la estética heterogénea e irrupción en las dinámicas sociales de los barrios periféricos. En I. Hernández García (ed.), Poéticas y criticas del devenir. Pensamiento de frontera sobre las formas de habitar (pp. 217236). Bogotá: Pontificia Universidad Javeriana.

Roy, A. (2009). The 21st-Century Metropolis: New Geographies of Theory. Regional Studies, 43(6), 819-830.

Ruipérez Palmero, R. (2006). ¿Quién teme a los pobladores? vigencia y actualización del housing by people de John Turner frente a la problemática actual del hábitat popular en América Latina. Bogotá: Universidad Nacional de Colombia.

Santos, M. (2000). La naturaleza del espacio. Barcelona: Ariel Geografía.

Sarria Icaza, A. y Tiriba, L. (9-11 de julio de 2003). Economia popular: conceptuando antiguas y nuevas prácticas sociales. Ponencia presentada en el simposio Los caminos recorridos por la economía popular solidaria, Montevideo, Asociación uruguaya de Historia Económica (Audhe).

Wagner, J. (2007). Observing Culture and Social Life, Documentary Photgraphy, Fieldwork and Social Resaearch. En G. C. Stanczak (ed.), Visual Research Methods, Image, Society and Representation (pp. 1-28). Londres: Sage.

Ward, P. (2004). Introduction and Overview: Marginality Then and Now. Latin American Research Overview, 39(1), 183-187.

Zibechi, R. (2008). América Latina: Periferias urbanas, territorios en resistencia. Bogotá: Ediciones desde abajo. 